\title{
Comparative Study on Flow Characteristics of Pipeline System Based on CFD
}

\author{
Hou Yingzhe ${ }^{1}$, Wu Hao ${ }^{1}$, Yan Xiaozhe ${ }^{1}$, Zhu Haoqiang ${ }^{1}$, Gao Haitao ${ }^{1}$, Hu Yiming ${ }^{1}$, Cui Qiguan ${ }^{1}$, Xiong Jinying ${ }^{1}$, \\ Zhou Longyu ${ }^{1}$
}

Wuhan Secondary Ship Design and Research Institute, Wuhan, Hubei, 430205, China

\begin{abstract}
Based on the CFD numerical simulation method, this paper established a pipeline model to study the internal flow characteristics of three different combinations of hot water pipeline systems and thus obtained the flow field information such as pressure, flow rate, and flow rate inside the pipeline. The study results showed that the smoother the pipeline transition, the smaller the velocity uniformity coefficient; the higher the uniformity of the flow field, the smaller the pressure and velocity fluctuations, the smaller the resulting pipeline vibration. Besides, the flow characteristics have been greatly improved.
\end{abstract}

\section{INTRODUCTION}

The hot water pipeline system is widely used in energy, shipbuilding, petrochemical, machinery, and other industries. It is the bridge and link connecting boilers, heat accumulators, heat exchangers, and other equipment in the power system. If the pipeline's design and layout are unreasonable, its resistance loss will be too large or too small. Too much resistance will cause the medium to flow poorly, and too small resistance will cause the medium to flow excessively and fastly, thus causing the pipeline to vibrate and affect the system's safety and reliability. Meanwhile, there are many pipelines, a variety of specifications, and complex arrangements in a large pipeline network. However, the pipeline network's arrangement and direction have significant limitations due to the limited space. Therefore, the pipeline network design should not only complete the arrangement of the pipeline system in the limited space, but also ensure that the parameters of each piece of equipment can reach the requirements of stable operation during running.

This paper studied the flow characteristics of the valve test system matched with the action performance test and discharge test of the regulator safety valve, analyzed three different pipeline combinations to improve the flow characteristics, and proposed an optimization scheme of the pipeline system combination.

\section{STRUCTURE PRINCIPLE PERFORMANCE PARAMETERS}

AND

\subsection{Structure principle}

The hot water test circuit is mainly composed of a $19 \mathrm{~m}^{3}$ heat accumulator, a $5 \mathrm{~m}^{3}$ hot water container, valves, pipelines, and accessories, as shown in Fig. 1.

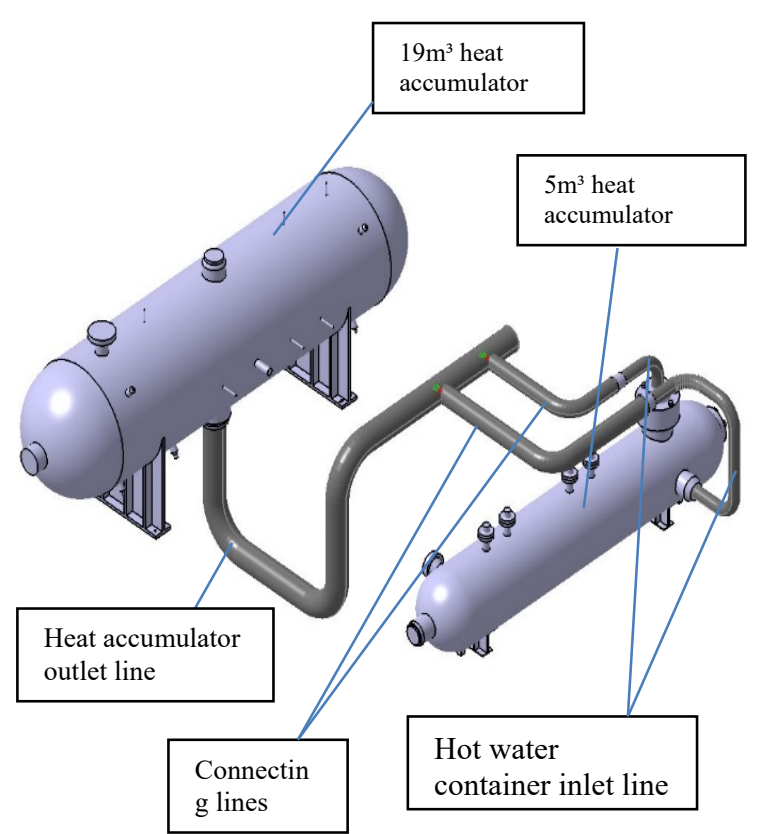

1. $19 \mathrm{~m}^{3}$ heat accumulator $2.5 \mathrm{~m}^{3}$ hot water container

3. Heat accumulator outlet line 4 . Connecting lines

5. Hot water container inlet line

Figure 1. Schematic Diagram of Hot Water Pipeline System Structure

\subsection{Performance Parameters}

Parameters of Hot Water Pipeline System (Table 1):

Table 1. Hot Water Pipeline Parameters

\begin{tabular}{|c|c|}
\hline Description & Value \\
\hline Heat accumulator volume & $19 \mathrm{~m}^{3}$ \\
\hline $\begin{array}{c}\text { Hot water container } \\
\text { volume }\end{array}$ & $5 \mathrm{~m}^{3}$ \\
\hline $\begin{array}{c}\text { Maximum test pressure } \\
(\mathrm{MPa})\end{array}$ & 20 \\
\hline
\end{tabular}

\footnotetext{
* Corresponding author: 15172526878@163.com
} 


\begin{tabular}{|c|c|}
\hline Temperature Range $\left({ }^{\circ} \mathrm{C}\right)$ & $333 \sim 343$ \\
\hline Medium & Saturated/supercooled water \\
\hline Heat accumulator outlet & $\varphi 406 \times 25$ \\
line & $\varphi 273 \times 34 / \varphi 406 \times 25 / \varphi 406 \times 50$ \\
Connecting lines \\
Hot water container inlet \\
line
\end{tabular}

In this paper, a comparative analysis is carried out on the specifications of two connecting pipelines in the pipeline system from a $19 \mathrm{~m}^{3}$ heat accumulator to a $5 \mathrm{~m}^{3}$ hot water container. The specific system pipeline combination is as follows:

1) Scheme A:

Heat accumulator outlet line $\varphi 406 \times 25$

Connecting lines $\varphi 273 \times 34$

Hot water container inlet line $\varphi 273 \times 34$

2) Scheme B:

Heat accumulator outlet line $\varphi 406 \times 25$

Connecting lines $\varphi 406 \times 25$

Hot water container inlet line $\varphi 273 \times 34$

3) Scheme C:

Heat accumulator outlet line $\varphi 406 \times 25$

Connecting lines $\varphi 406 \times 50$

Hot water container inlet line $\varphi 273 \times 34$

\section{Numerical Simulation}

\subsection{Governing equation}

Captions should be typed in 9-point Times. They should be centred above the tables and flush left beneath the figures.

In this paper, the mature standard model was used for numerical simulation, and the governing equations were composed of continuity equation, momentum conservation equation, turbulent kinetic energy $\mathrm{K}$ equation, and dissipation energy equation.

Continuity equation

$$
\text { - } \frac{\partial \rho}{\partial t}+\frac{\partial}{\partial x_{i}}\left(\rho u_{i}\right)=0
$$

Momentum conservation equation (N-S equation) formula

$$
\begin{aligned}
& \rho \frac{d u}{d t}=\frac{\partial p}{\partial x_{i}}+\frac{\partial}{\partial x_{j}}\left[\mu\left(\frac{\partial u_{i}}{\partial x_{j}}+\frac{\partial u_{j}}{\partial x_{i}}-\frac{2}{3} \delta_{i j} \frac{\partial u_{i}}{\partial x_{j}}\right)\right] \\
& +\frac{\partial}{\partial x_{j}}\left(-\overline{u_{i}^{\prime} u_{j}^{\prime}}\right)
\end{aligned}
$$

The transport equations $\varepsilon$ of turbulent kinetic energy $\mathrm{k}$ and turbulent kinetic energy dissipation rate of the standard turbulence model can be expressed as:

$$
\frac{\partial(\rho k)}{\partial t}+\frac{\left(\partial \rho k u_{i}\right)}{\partial x_{i}}=\frac{\partial}{\partial x_{i}}\left[\left(\mu+\frac{\mu_{t}}{\delta_{k}}\right) \frac{\partial k}{\partial x_{j}}\right]+P-\rho \varepsilon
$$

$$
\frac{(\partial \rho \varepsilon)}{\partial t}+\frac{\left(\partial \rho \varepsilon u_{i}\right)}{\partial x_{i}}=\frac{\partial}{\partial x_{j}} \mu+\frac{\mu_{t}}{\sigma_{Z}} \frac{\partial \varepsilon}{\partial x_{j}}+\frac{\varepsilon}{k}\left(C_{\varepsilon 1} P+\rho C_{\varepsilon 2} \varepsilon\right)
$$

Where $u_{i}$ - the velocity component in the direction i, $\mathrm{i}=1,2,3$

$\mu$ - Kinematic viscosity coefficient of the medium

$u_{t}$ - Eddy viscosity coefficient

$\delta_{k}$ - Prandtl number corresponding to turbulent kinetic energy $\mathrm{k}$

$\delta_{\varepsilon}$ - Prandtl number corresponding to turbulent kinetic energy dissipation rate $\varepsilon$

$\mathrm{P}$ - turbulent kinetic energy generation term

$$
P=\mu_{t}\left(\frac{\partial u_{i}}{\partial x_{j}}+\frac{\partial u_{j}}{\partial x_{i}}\right) \frac{\partial u_{i}}{\partial x_{j}}
$$

wherein:

$$
\mu_{t}=C_{\mu} \rho \frac{k^{2}}{\varepsilon}
$$

$\mathrm{k}-\varepsilon$ Empirical constants in the model (Table 2):

Table 2. Coefficient in k- $\varepsilon$ Model

\begin{tabular}{ccccc}
\hline$C_{\varepsilon 1}$ & $C_{\varepsilon 2}$ & $C_{\mu}$ & $\delta_{k}$ & $\delta_{\varepsilon}$ \\
\hline 1.44 & 1.92 & 0.09 & 1.0 & 1.3 \\
\hline
\end{tabular}

\subsection{Establishment of Flow Channel Model and Meshing}

According to the specific structure parameters, the 3D model of the pipeline system is established by CATIA software, and the internal flow channel model is generated by reverse modeling of fluid mechanics software. The flow channel model is imported into ANSYS ICEM CFD for meshing (Fig. 2). The mesh for numerical calculation is divided by tetrahedral/ hybrid mesh, and the flow channel corners are locally encrypted to make the calculation results more accurate, and the mesh independence test is performed.

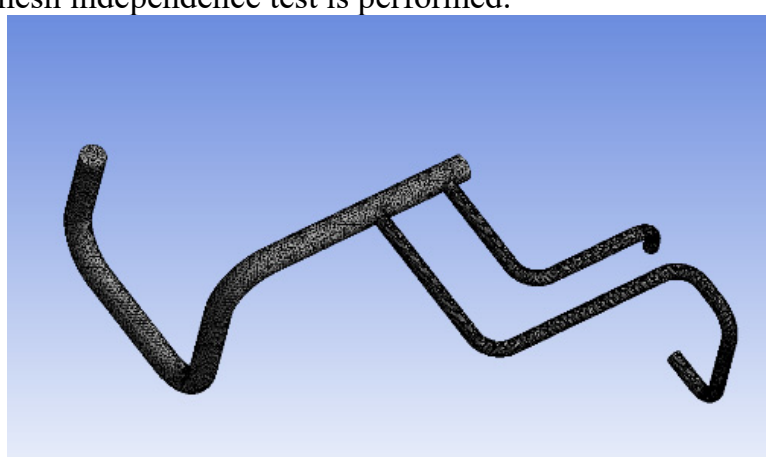

a) Scheme $A$ 


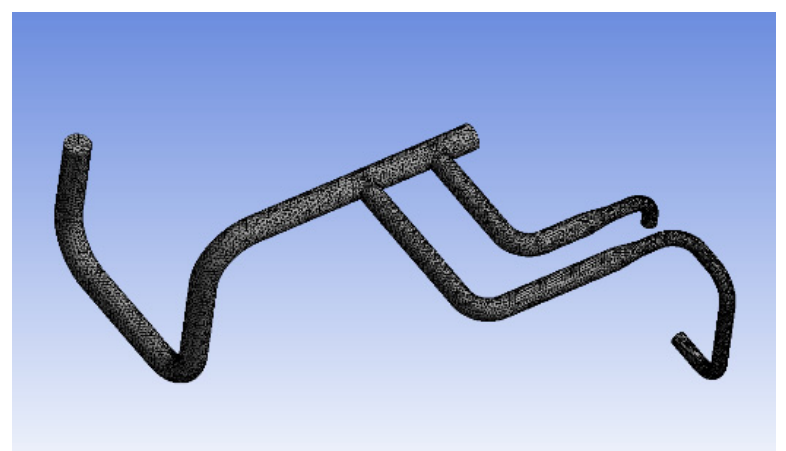

b) Scheme B

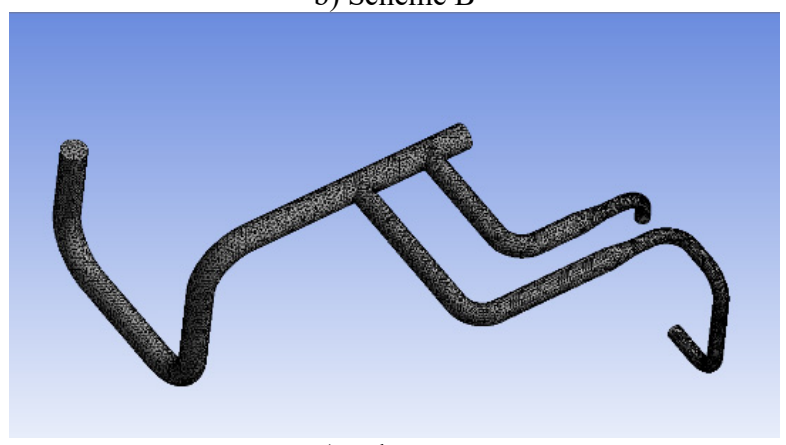

c) Scheme $\mathrm{C}$

Figure 2. Mesh Model of the Pipeline System

Combined with the running state of the hot water pipeline system in actual working conditions, the internal flow model under typical working conditions is established. The 3D incompressible flow Reynolds timeaveraged equations and k- $\varepsilon$ turbulence model are used to form a closed equation set to solve the internal flow field of the pipeline system. The fluid medium is set to water, and the temperature change is not considered during the solution process. The heat accumulator outlet is set as the pressure inlet boundary condition, with a velocity of 19.5 MPa. The inlet 1 and 2 of the hot water container are set as the pressure outlet boundary condition of 17.2 $\mathrm{MPa}$. The pipe wall is set as the adiabatic non-slip boundary condition, and the $3 \mathrm{D}$ flow field is numerically simulated and analyzed.

\section{Study on Internal Flow Characteristics}

\subsection{Pressure Field Distribution}

Fig. 3 is a pressure nephogram of different pipeline combinations. As shown from the figure, Scheme A's pressure gradient changes significantly, with uneven pressure distribution and a maximum pressure appearance at the end of the pipeline. That of Scheme B is small, with a smooth transition of pressure fluctuation and relatively uniform distribution. Compared with Schemes A and B, Scheme C has the smoothest pressure fluctuation and the most optimized flow characteristics.

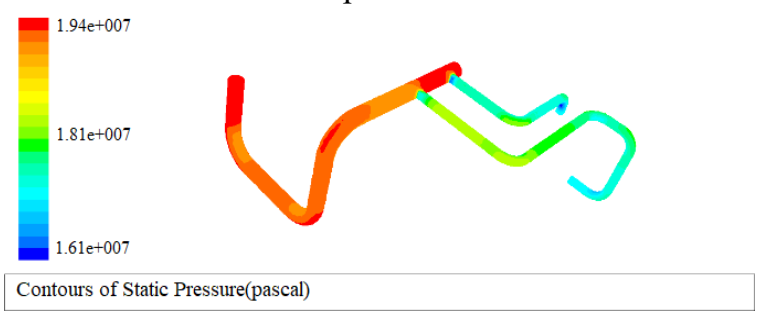

a) Scheme $A$

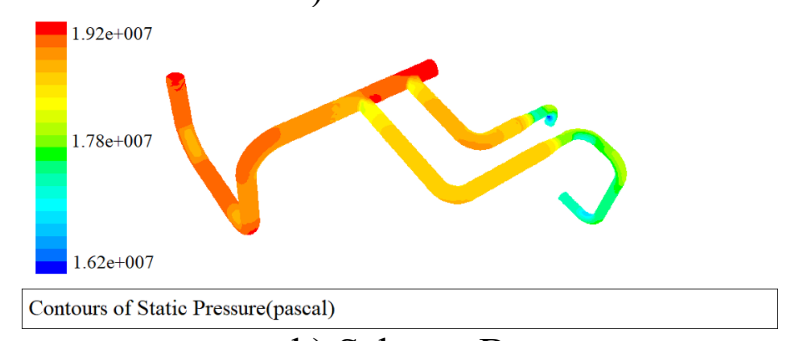

b) Scheme B

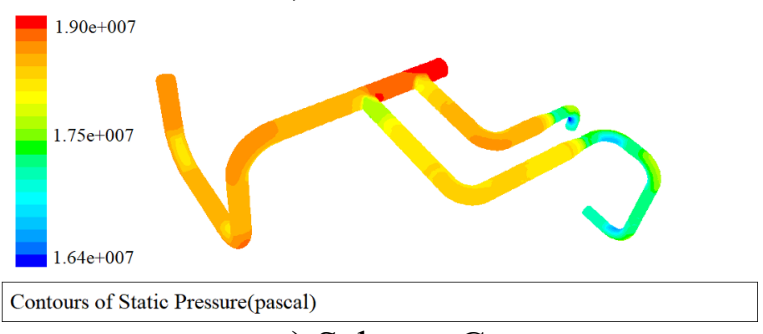

c) Scheme C

Figure 3. Pressure Nephogram of Different Pipeline Combinations

\subsection{Velocity field distribution}

A velocity distribution nephogram is obtained after study on the velocity field distribution of different pipeline combinations, as shown in Fig. 4. As shown from the figure, Scheme A's flow rate is relatively high, with significant velocity gradient changes and maximum velocity appearance at the junction of the outlet pipeline of the heat accumulator and the connecting pipeline, causing certain scouring to the pipeline. Scheme B's maximum flow rate is generated at the hot water container inlet, with a relatively gentle velocity fluctuation and a low flow rate area formed at the connecting pipeline compared with other schemes. Compared with Schemes A and B, Scheme C gradually changes in transition, with uniform velocity distribution, stable tends, and optimized flow characteristics.

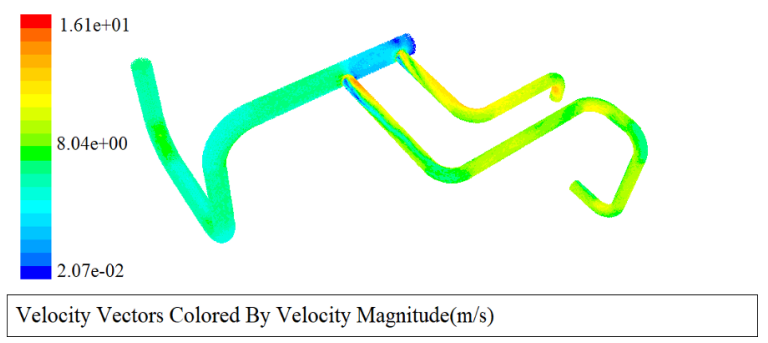

a) Scheme A

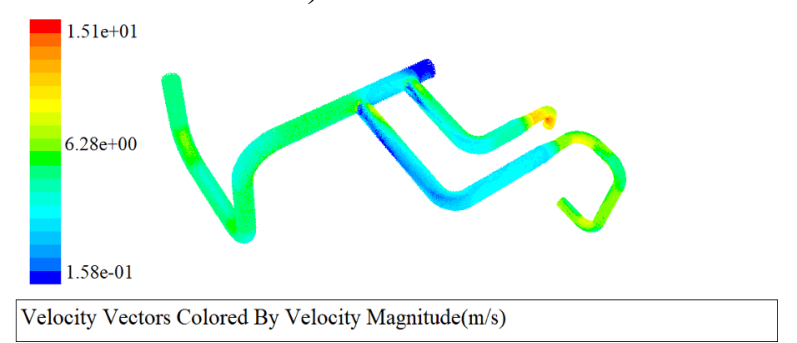

b) Scheme B 


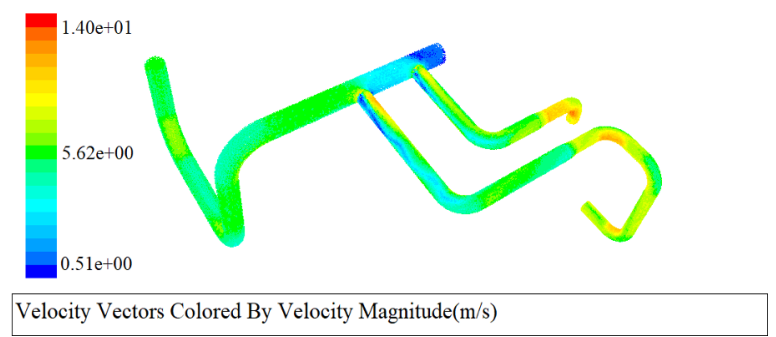

c) Scheme C

Figure 4. Velocity Nephogram of Different Pipeline Combinations

\section{Conclusion}

(1) Scheme $\mathrm{C}$ adopts $\varphi 406 \times 50$ connecting pipelines, and the variation law of pressure gradient and velocity gradient tends to be more stable than Schemes A and B.

(2) The smoother the pipeline combination transition, the higher the uniformity of the flow field, the smaller the pressure and velocity fluctuations, the smaller the pipeline vibration, and the flow characteristics will be greatly improved.

\section{References}

1. Yang Xueyin. Flow Analysis and Optimal Design of Typical Pipeline Accessories [D]. Harbin Institute of Technology, 2012.7.

2. Chi Ting. Hydraulic Calculation of Steam Pipeline and Study on Flow Field Characteristics of Valve Transient Closure [D]. Harbin Engineering University, 2015.3.

3. Wang Huihui. Study on Flow Characteristics and Noise of Pipeline System [D]. Huazhong University of Science and Technology, 2017.5.

4. Wang Zeshen. Fluid-structure Coupling Vibration Test and Numerical Simulation of Water Transmission Pipeline [D]. Harbin Institute of Technology, 2016.6.

5. Wang Daobin, Zhao Wenli, et al. Analysis of Flow Resistance Characteristics and Structure Optimization of Brake Pipeline Based on FLUENT [J]. Journal of Mechanical \& Electrical Engineering, 2012, 29 (10): 1172-1174.

6. Gao Lufeng, Zheng Haicun. A Hydraulic-Thermal Coupling Calculation Method of Steam Pipe Network [J]. China Sciencepaper, 2013, 08: 812-815.

7. Wang Hai. An Object-oriented Hydraulic Calculation Method of Multi-heat Source Annular Pipe Network [N]. Journal of Zhejiang University (Engineering Science), 2012, 46 (10). 